TRABAJO EN PROGRESO

\title{
Desarrollo de un análisis contextual para mejorar la usabilidad en sitios Web: Caso práctico herbario digital
}

\author{
Alma Delia Olmos Pantoja, María del Rosario \\ Peralta Calvo, Zulma Janet Hernández Paxtián, \\ Rocío Rosas López
}

Publicado: 31 Octubre 2018

\begin{abstract}
Resumen
La usabilidad es un término que ha tomado cada vez mayor importancia en el desarrollo de software. No es una tarea sencilla, lograr que se cumplan con las tareas requeridas en un contexto determinado de usuarios. En el presente trabajo partimos de la siguiente pregunta: ¿Cómo lograr un mayor grado de usabilidad en sitios web aplicando un análisis contextual? Para contestarla se plantea el caso práctico de un herbario digital, con la realización de un análisis contextual identificando los posibles usuarios y sus perfiles, obteniendo la información que espera encontrar en el herbario. Lo anterior bajo el enfoque del Diseño Centrado en el Usuario (DCU) y como un paso previo a la conformación de la arquitectura de la información del herbario digital.
\end{abstract}

Palabras clave: Análisis contextual; Usabilidad; Diseño centrado en el usuario, Interacción Humano - Computadora.

\section{Introducción}

Cada día se integran a la red miles de sitios web, algunos son visitados por millones de usuarios, mientras que otros pasan desapercibidos, esto se puede deber a diversas causas: como la accesibilidad, el grado de interés del contenido, el posicionamiento en que son situados en el buscador, o su grado de usabilidad [3]. Ésta última, es definida como el rango en el cual un producto puede ser usado por un grupo de usuarios específicos para alcanzar ciertas metas definidas con efectividad, eficiencia y satisfacción en un contexto de uso especificado [2]. Existen diversos métodos y técnicas de evaluación de la usabilidad, como los de indagación que consiste en hablar con los usuarios y observarlos detenidamente usando el sistema en trabajo real y obteniendo respuestas a preguntas formuladas verbalmente o por escrito [4]. Un ejemplo de método de indagación es el análisis contextual.

El presente trabajo se centra en desarrollar un análisis contextual, para obtener información sobre el contexto de uso, en

\footnotetext{
Olmos Pantoja, AD., Peralta Calvo, MR., Hernández Paxtián, ZJ., Rosas

López, R.

Universidad de la Cañada

Carretera Teotitlán - San Antonio Nanahuatipan Km. 1.7 s/n. Paraje

Titlacuatitla. Teotitlán de Flores Magón, Oaxaca, México.

Email: 1i_almapantoja@unca.edu.mx,mperalta@unca.edu.mx,

jpaxtian@unca.edu.mx,rocio@unca.edu.mx
}

donde los usuarios son entrevistados y observados en su propio medio ambiente, forma parte de la fase de estudio del enfoque DCU, en el diseño de sistemas interactivos. Se opta por seguir el DCU porque persigue obtener información sobre los usuarios, sus tareas y sus objetivos, y utilizar la información obtenida para orientar el diseño y desarrollo de los productos [5], y poder obtener sitios web más usables que cumplan con los requerimientos de los usuarios y se incorpore fácilmente a su contexto.

\section{Estudio}

Al realizar una búsqueda de sitios web existentes, en especial los dedicados a herbarios digitales, se observó: que el usuario no logra realizar tareas de forma eficiente y efectiva, no cuenta con una consistencia en el diseño de las interfaces, en consecuencia, resalta la necesidad de tomar en cuenta a los usuarios y su contexto [1]. Durante la realización del análisis contextual es necesaria una planeación que consiste en la identificación de los temas a abordar, los objetivos, así como las localidades a abarcar y los perfiles de usuarios identificados.

Una vez definido lo anterior, se procede a la ejecución del análisis contextual. Se inició con el recorrido por las comunidades, percatándose de la gran riqueza cultural, flora, fauna y gastronomía, que sin duda definirían las particularidades de los perfiles de usuario, sobre todo de los médicos tradicionales. El contexto de los médicos tradicionales, está definido por un conocimiento de generaciones ancestrales que se ha fortalecido por la necesidad de dar cura a problemas de salud con las plantas medicinales. Este sector se ha organizado para contar con permisos, poder operar en sus comunidades y ser reconocidos. Además, de capacitarse con apoyo de la comunidad científica.

El acceso a conocimiento de plantas por el momento ha sido sólo las que están a su alcance y de manera muy rústica, es decir, ellos hacen su colecta y anotaciones para lo que sirven éstas. Con respecto al uso de la tecnología, básicamente la computadora y el acceso a internet no se ha dado, porque sólo acceden a la información de las plantas de su comunidad. Por otro lado, el contexto de alumnos y profesores está definido por el ambiente universitario, con las actividades que realizan en clases con asignaturas donde las tareas consisten en consultar información de plantas de la región donde se encuentran, para posteriormente pasar por un proceso de estudio en áreas de fotoquímica, nutrición, agroindustrial, por mencionar algunas. En la siguiente 
sección se dan a conocer de forma más puntual los resultados obtenidos del análisis contextual.

\section{Resultados}

El análisis contextual constó en la realización de entrevistas a usuarios que cumplían con los perfiles definidos, posteriormente se apoyó en el método de Card Sort, que ayudó a la generación de una estructura de la información y sugerencias para la navegación, menús y taxonomía. Se realizaron entrevistas a alumnos, profesores investigadores y médicos tradicionales de la región, logrando un total de 15 entrevistados, de los cuales nueve participaron en el Card Sort (ver Tabla 1).

Tabla 1. Participantes en el análisis contextual desarrollado en la fase de estudio de un herbario digital $(M=$ mujeres y $\mathbf{H}=$ hombres)

\begin{tabular}{|l|c|c|}
\hline \multicolumn{1}{|c|}{ Perfil de usuario } & Entrevistas & Card Sort \\
\hline Alumnos & $2 \mathrm{M}+3 \mathrm{H}$ & $1 \mathrm{M}+2 \mathrm{H}$ \\
\hline Profesores investigadores & $1 \mathrm{M}+4 \mathrm{H}$ & $1 \mathrm{M}+4 \mathrm{H}$ \\
\hline Médicos tradicionales & $3 \mathrm{M}+2 \mathrm{H}$ & $1 \mathrm{M}$ \\
\hline Total & 15 & 9 \\
\hline
\end{tabular}

Cabe mencionar que todos los usuarios entrevistados tenían conocimiento previo sobre plantas medicinales, aunque algunos confundían el término de un herbario. Los alumnos expresaron interés en el herbario digital ya que opinan que este sería de gran utilidad para estudiantes del área. En la Tabla 2 se muestran los resultados más sobresalientes de la realización de las entrevistas.

En general los usuarios interesados en el uso de un recurso tecnológico para poder acceder a la información del herbario digital son los alumnos y profesores investigadores. Los médicos tradicionales lo consideran un reto, debido a la brecha digital que existe aún para el uso de la tecnología en su contexto. Por lo que, cubrir las necesidades de los médicos tradicionales, será uno de los mayores retos del herbario digital. Las entrevistas ayudaron a conocer algunas de las características o ítems que los usuarios identifican son con los que cuenta una planta dentro de un herbario, los cuales debían ser clasificados, es por ello que se recurre al método de Card Sort, el cual sirve para la creación de una estructura de la información, probables estructuras de navegación. Además de ayudar a identificar diferentes categorías.

Una vez finalizada la realización de las entrevistas y obtenidos los puntos más importantes en cuanto a la información de las plantas que los usuarios creen necesitar dentro del herbario digital, se procedió a realizar la preparación del Card Sort, con el fin de proporcionar una referencia al usuario para la información que considera viable puede ser candidata a estar en un herbario, se inició colocando en etiquetas las características de las plantas que los entrevistados mencionaron, además de utilizar como referencia diferentes colores para que los usuarios definieran los nombres de los encabezados de cada sección.

Los usuarios clasificaron la información de las plantas en secciones, a las cuales asignaron un nombre general que identificara al grupo de características, algunos usuarios participaron en equipo y otros individualmente, tal es el caso del médico tradicional en su aplicación (Figura 1).

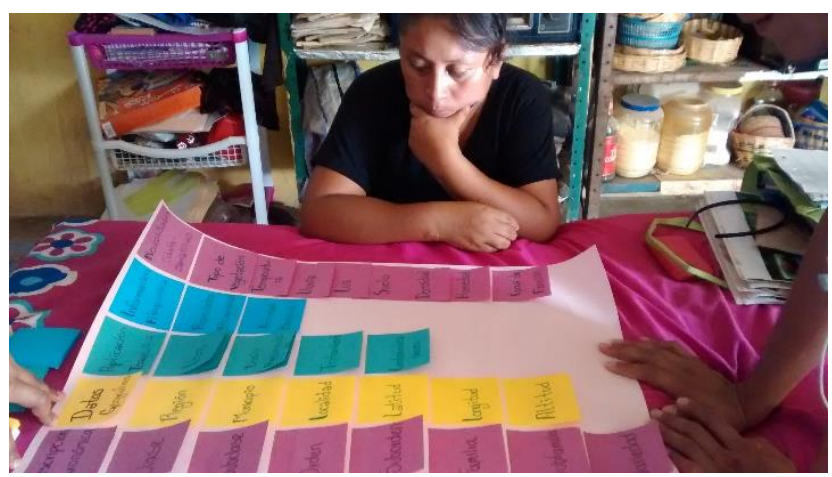

Figura 1. Aplicación del método de Card Sort a un usuario

Al finalizar la aplicación del método de Card Sort se reunieron las opiniones y la información obtenida de los tres perfiles de usuarios, dando como resultado una clasificación de los ítems identificados, estos agrupados en seis categorías, desde los datos generales, que son básicos para los tres perfiles de usuarios, hasta la información fitoquímica que les es útil conocer a los especializados en el área (Figura 2).

La aplicación del método de Card Sort ayudó a la identificación de nuevos ítems y a su clasificación en diferentes categorías, como son: datos generales, descripción taxonómica, datos geográficos, entre otros, los cuales serán de ayuda para los

Tabla 2. Resultados de las entrevistas de acuerdo a cada perfil de usuario

\begin{tabular}{|c|c|c|}
\hline Alumno & Profesor investigador & Médico tradicional \\
\hline \multicolumn{2}{|c|}{$\begin{array}{l}\text { Identifican a un herbario como una colección de plantas, con un conjunto de } \\
\text { características inherentes. }\end{array}$} & $\begin{array}{l}\text { Confunden lo que es un herbario y manejan } \\
\text { el término de plantas. }\end{array}$ \\
\hline \multicolumn{2}{|c|}{ Los entrevistados mencionaron haber utilizado algún herbario anteriormente. } & $\begin{array}{l}\text { Utilizan plantas para su labor pero no lo } \\
\text { identifican como un herbario. }\end{array}$ \\
\hline $\begin{array}{l}\text { Identifican las características generales de } \\
\text { una planta, tales como nombre común, } \\
\text { nombre científico, género, especie. }\end{array}$ & $\begin{array}{l}\text { Las principales características que } \\
\text { identifican en un herbario son: cédulas, } \\
\text { tarjetas, siempre cuentan con una } \\
\text { clasificación, género, especie, entre otras. }\end{array}$ & $\begin{array}{l}\text { Los médicos eran reconocidos dentro de las } \\
\text { organizaciones: "Médicos Indígenas de la } \\
\text { Cañada (OMIC)" y "María Sabina". }\end{array}$ \\
\hline $\begin{array}{l}\text { Por lo general recurren a un herbario para } \\
\text { consultar información acerca de plantas } \\
\text { medicinales. }\end{array}$ & $\begin{array}{l}\text { Les interesa conocer algunas características } \\
\text { de las plantas, ya sea por sus propiedades } \\
\text { fitoquímicas, o por su poder curativo. }\end{array}$ & $\begin{array}{l}\text { El utilizar la tecnología actual les representa } \\
\text { un reto ya que no están familiarizados con } \\
\text { su uso. }\end{array}$ \\
\hline $\begin{array}{l}\text { A los alumnos les interesaría tener un } \\
\text { herbario digital disponible de preferencia } \\
\text { en un dispositivo móvil. }\end{array}$ & $\begin{array}{l}\text { Les gustaría tener una herramienta donde } \\
\text { consultar la información de las plantas que } \\
\text { se encuentran dentro de la Región Cañada. }\end{array}$ & $\begin{array}{l}\text { El comunicarse con los médicos } \\
\text { tradicionales representó un reto ya que estos } \\
\text { hablan su lengua materna. }\end{array}$ \\
\hline
\end{tabular}


diferentes perfiles de usuarios que deseen realizar una consulta en el herbario digital. Los datos generales y la aplicación terapéutica suelen ser datos que normalmente les interese consultar a un usuario no familiarizado con tecnicismos del área de especialización sobre las plantas, en cambio para los más avanzados les podría interesar consultar alguna planta para conocer sus propiedades fitoquímicas, su descripción taxonómica o en el caso del área agropecuaria, las necesidades edafoclimáticas de dicha planta.

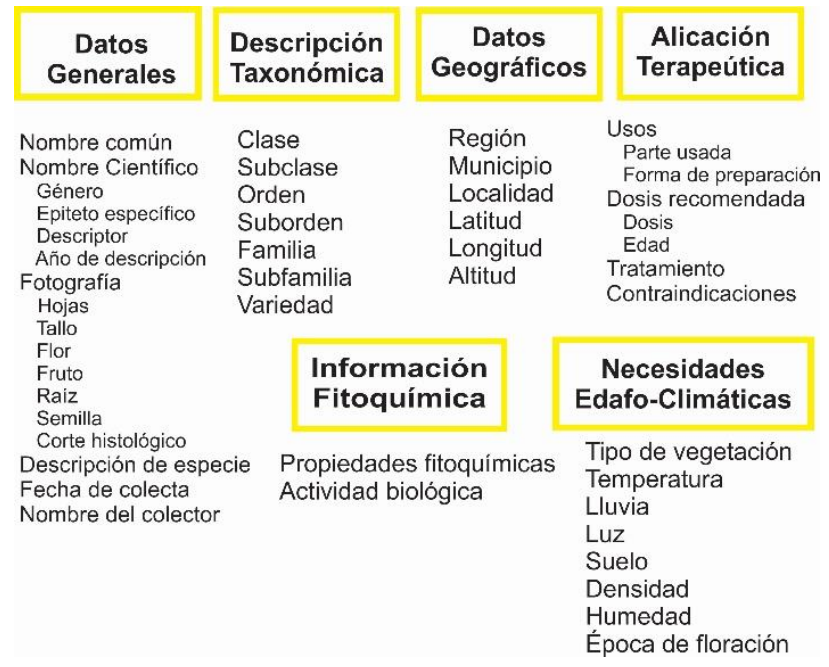

Figura 2. Clasificación de los ítems de acuerdo a categorías definidas por los participantes.

\section{Conclusiones}

La primera fase de DCU entregó información del usuario a las siguientes fases, permitiendo que el diseño y la implementación cuenten con elementos necesarios para poder ofrecer un sistema interactivo usable a los perfiles de usuario ya definidos. También, en este trabajo se evidencia el correcto uso de técnicas como las entrevistas, la observación y card sort, de otra manera no hubiera sido posible la obtención de resultados en el análisis contextual. La realización del análisis contextual encamina a la obtención de usabilidad en el herbario digital, se logra conocer a los usuarios específicos en su contexto específico.
Entre las ventajas de haber utilizado el método Card Sorting es que es simple y rápido de utilizar, involucra a los usuarios. Además de ser muy económico ya que solo se necesita contar con algunas tarjetas y marcadores para anotar los ítems a clasificar. Algunas de las desventajas a considerar es que no considera las tareas que los usuarios realizan, únicamente a la clasificación de la información, los resultados son variables y su análisis requiere un tiempo considerable.

El haber realizado el análisis contextual ayudará al trabajo a futuro, que es la construcción de la arquitectura de la información de acuerdo a los resultados obtenidos y dar continuidad a las siguientes fases del enfoque DCU, hasta llegar a la implementación del herbario digital.

\section{Agradecimientos}

Agradecemos a todos los participantes en el análisis contextual, al personal de soporte y publicaciones, quienes escribieron y proporcionaron comentarios útiles sobre versiones anteriores de este documento. Además, los autores agradecen la concesión de NSF (\# 1234-2012-ABC).

\section{Referencias}

[1] Crisantos L. Peralta, M. y Hernández, Z. Evaluación Heurística a Interfaces Gráficas de Usuario de Herbarios Digitales. MexIHC 2014.

[2] ISO 9241-11. Ergonomic Requirements for office work with visual display terminals (VDTs) - part 11: Guidance on Usability. International Organization for Standardization. 1998.

[3] Pérez, M. Arquitectura de la información en entornos web. El profesional de la información. Rev 19 (2010), 4: 333-337.

[4] Perurena, L. and Moráguez, M. Usabilidad de los sitios web, los métodos y las técnicas para la evaluación. Revista Cubana de Información en Ciencias de la Salud. Rev 24 (2013), 2: 176-194.

[5] Rubin, J. Handbook of usability testing. Wiley \& Sons, Inc. New York., 1994. 